\title{
Unplugged Computing for Children
}

\author{
Maria Alandes Pradillo ${ }^{1}$, Eszter Badinova ${ }^{1}$, Anda-Catalina Chelba ${ }^{1}$, Miguel Hermo Serans $^{1}$, \\ Natalie Kane ${ }^{1}$, Simona Kriva ${ }^{1}$, and Hannah Short ${ }^{1, *}$ \\ ${ }^{1}$ European Organization for Nuclear Research (CERN)
}

\begin{abstract}
The number of women in technical and computing roles in the High Energy Physics (HEP) community hovers at around $15 \%$. At the same time there is a growing body of research to suggest that diversity, in all its forms, brings positive impact on productivity and well-being. These aspects are directly in line with many organisations' values and missions, including CERN. Although proactive efforts to recruit more women in our organisations and institutes may help, the percentage of female applicants in candidate pools is similarly low and limits the potential for change. Factors influencing the career choice of girls have been identified to start as early as primary school and are closely tied to encouragement and exposure. It is the hope of various groups in the HEP community that, by intervening early, there may be a change in demographics over the years to come. During 2019, the Women in Technology Community at CERN developed two workshops for 6-9 year olds, which make the fundamental concepts of ICT and Computer Science accessible to young people with no prior experience and minimal assumed background knowledge. The immediate objectives were to demystify computer science, and to allow the children to meet a diverse set of role models from technical fields through our volunteer tutors. The workshops were run multiple times over 2019. This paper contains an overview of our motivation, describes the content of the workshops, results, lessons learnt and the future evolution of such activities.
\end{abstract}

\section{Introduction}

The percentage of women in professional Information Computer Technology (ICT) positions stands at approximately $20 \%$ in most western countries, with that percentage dropping as low as $10 \%$ for roles involving hardware [1]. In the HEP Community, we regularly see this figure standing at roughly $15 \%$ [2]. At each stage of education, the number of female ICT students decreases leaving a dwindling candidate pool for an ever-expanding job market. Multiple factors influence girls' decisions to focus their careers elsewhere, principally influenced by lack of encouragement from available role models and cultural perceptions of the attractiveness of jobs in ICT [1][3]. At the same time, there is a growing body of evidence to suggest that diverse teams perform better [4], and that software does not reflect the needs of the human population without the input of women [5]. As one of its objectives, the Women in Technology Community [6] at CERN aims to contribute to redressing the lack of gender diversity in technical fields.

\footnotetext{
*e-mail: hannah.short@cern.ch
} 


\subsection{Women in Technology Community at CERN}

CERN's Women in Technology Community (WIT) [6] was formed in 2016 to create a fairer working environment that gives women equal opportunities to feel empowered to progress in technical fields for the benefit of Science and Technology. WIT's main objectives are: networking for help, support and career; increasing awareness of gender issues; and helping promote diversity and equality. WIT is an open community and actively welcomes participation from all, regardless of gender or technical persuasion. It is organised by a Steering Committee, whose members change regularly, and activities are announced on a mailing list, wit-matters@cern.ch.

WIT offers a range of activities such as:

- Weekly emails: full of interesting links from the week's news and research concerning women in STEM subjects. Some serious, some light-hearted, always interesting and definitely thought-provoking.

- Interviews: with women in leading roles at CERN, in industry or in academia about their careers and backgrounds, giving them the chance to inspire fellow women and share their views on how to improve the gender balance.

- Diversity talks: with senior male colleagues who talk around their experiences and thoughts on gender diversity and gender balance, and why this has been important to them both professionally and personally.

- Mentoring: there is a yearly mentoring program open to everyone.

- Networking events: visits to different areas at CERN are organised to help widen horizons, followed by a networking session to promote collaboration.

- Outreach: the WIT community participate in events in the local community to teach programming and computing basics.

- Annual Review: the Steering Committee prepares a summary of the previous year and invites input from the Community on future activities.

Since the outset, outreach was an activity of interest to many of its members, particularly focusing on inspiring women to develop an interest in ICT. WIT has held multiple outreach events for girls, boys and adults, teaching fundamental concepts of Computer Science. Women and men of many nationalities express interest to tutor these outreach activities and this diverse volunteer pool provides participants with a source of role models.

In 2019, WIT ran its first "Unplugged" workshops - workshops that do not require a computer. Details of all material curated by WIT, as well as links to external resources, can be found at cern.ch/wit/outreach.

\subsection{Motivation for Unplugged Workshops}

During the past years, WIT has been frequently approached to provide computing outreach. Requests have come from parents keen to push their children into the field, from external organisations, and from technical members of the CERN community who are motivated to encourage the next generation themselves. There is a clear demand for such content.

Unplugged workshops have several key benefits over workshops requiring computers:

- bringing computers adds logistical difficulty, cost and distraction;

- not all children are familiar with computers, which present a huge additional learning curve for some; 
- the format enables a high degree of interaction between tutors and participants, where tutors can ensure that participants are engaged and learning;

- many parents express a wish to limit screen-time for their children.

\section{Method}

A meeting was held in late 2018 to brainstorm ideas for Unplugged workshops. This meeting came up with two main concepts to develop; programming basics (logic, conditionals, loops), and demystifying computers (understanding basic hardware terms). Once the themes were decided, two projects were kicked off in parallel. The leaders identified existing material that could be reused and modified to fit the initial workshop length (1.5 hours), and translated to French.

Once the material was finalised, the WIT community was called upon to volunteer as tutors and attend a training. Several iterations of the material were produced based on feedback during those training sessions. Male and female volunteers were welcome.

The first event was held in Spring 2019 during Geneva's Equality Week, in collaboration with RightsTech Women, a Swiss, non-profit association dedicated to advancing the human rights of women and girls in the fields of science, technology, engineering and mathematics (STEM) [7]. Both workshops, now called "Introduction to Programming" and "What's Inside a Computer?", were run twice. The workshops were repeated several times during the year.

At all stages in workshop preparation, care was taken to include the WIT Community and collaborate with other relevant groups in order to create an open and transparent atmosphere.

\subsection{Introduction to Programming (for ages 6-9)}

The "Introduction to Programming" workshop is based on "1,2,3 Codez" [9], a French project to introduce students and teachers to computer science. The project is supported by the foundation La main à la pâte, created in 2011 by the Académie des sciences and the Ecoles normales supérieures de Paris et de Lyon.

A grid, maze and arrow cards are used by children to navigate a robot between two squares. The game is extended by including boxes that must be opened (using a new instruction card), and then only opened on certain conditions. Participants take a booklet home containing further exercises.

\subsection{What's inside a Computer? (for ages 6-9)}

The "What's inside a Computer" workshop is inspired by Hello Ruby Tutorial [8], "My First Computer". Participants learn about some core components of computers (memory, hard drive, graphical processor), and complete a role playing game in which an image must be retrieved from the memory and then output (drawn) onto paper. The participants then build a paper model of a computer, which can be taken home. 


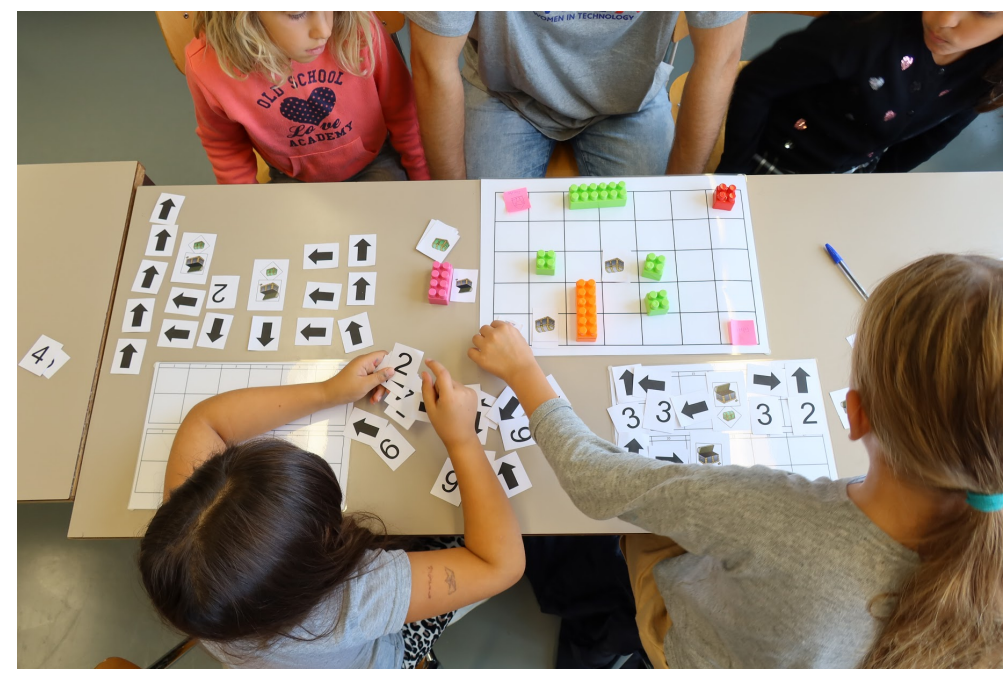

Figure 1. "Introduction to Programming" workshop in action

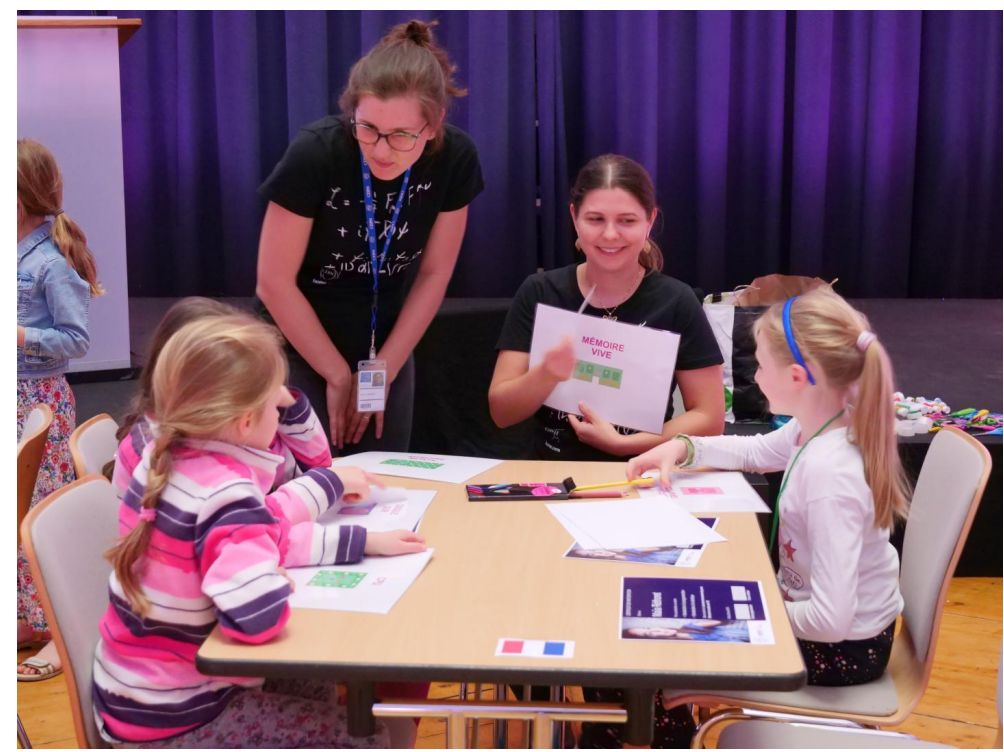

Figure 2. "What's inside a Computer?" workshop in action

\section{Participants}

During 2019, the Unplugged Workshops were completed by close to 500 children. Three events were held specifically for girls, and CERN's Open Days provided an opportunity to extend it to boys. Workshops were run in both English and French at each event. 


\begin{tabular}{llll} 
Date (2019) & Event Name & $\begin{array}{l}\text { Approximate } \\
\text { Participants }\end{array}$ & Volunteers \\
\hline \hline March 9 & Semaine de L'Egalité, & 80 & 13 \\
& HEPIA, Geneva & & 18 \\
April 25 & Girls in ICT Day, CERN & 50 & 18 \\
September 14-15 & CERN Open Days, CERN & 250 & 9 \\
October 5 & EU Code Week, HEPIA, 80 & 9 \\
& Geneva & &
\end{tabular}

\section{Lessons learnt}

The workshops improved each time they were run, with tutors being increasingly able to maintain the children's engagement for the duration of the session. Thorough training for tutors was found to be highly important, with an effort made to encourage volunteers to attend multiple events. Conversely, volunteering represents a significant time investment small incentives (such as T-Shirts) were used to ensure sufficient sign-up.

In terms of participants, we learned that there is a wide variation in computer literacy, stemming from differences in age, school curricula and parental engagement. Tutors needed to be flexible and adapt to the children's level. Children often wish to continue at home and effort was spent in creating material that could be taken away at the end of the workshop. We found it more effective to attempt to group children by similar ages, whenever possible, as it was observed that age difference can have a detrimental effect on engagement and confidence. In addition, interested children return to the workshops and there is a persistent need for new material.

\section{Conclusion}

Unplugged Workshops have been an effective way to engage children in ICT fundamentals, with limited logistical difficulty or cost. During 2019, CERN's Women in Technology Community (WIT) tutored close to 500 children. Of these children a large proportion were female; it is hoped that by providing a fun activity, run by role models that encourage their interest, we may influence some of them to take up technical careers.

WIT's outreach activities will continue to evolve in the coming years, with emphasis being placed on making content reusable. There is interest to provide Unplugged Workshops for both younger and older age groups. It is hoped that CERN's Science Gateway [10] will provide a sustainable location for these workshops, and an opportunity to create a syllabus to continue engagement with local children. We are fortunate to operate in an environment with a mission statement that highlights education and inspiration [14], and are grateful for the support that we receive.

\section{A WIT Outreach Material}

All outreach material can be found at cern.ch/wit/outreach. We include here a brief description of the existing workshops not covered by this paper, with the hope that it may prove useful to others.

\section{A.1 Introduction to Programming (for ages 10-14)}

For the more advanced programming workshop, we combine web-based programming with an interactive game. Participants start in pairs - one is a robot and one is a programmer. The 

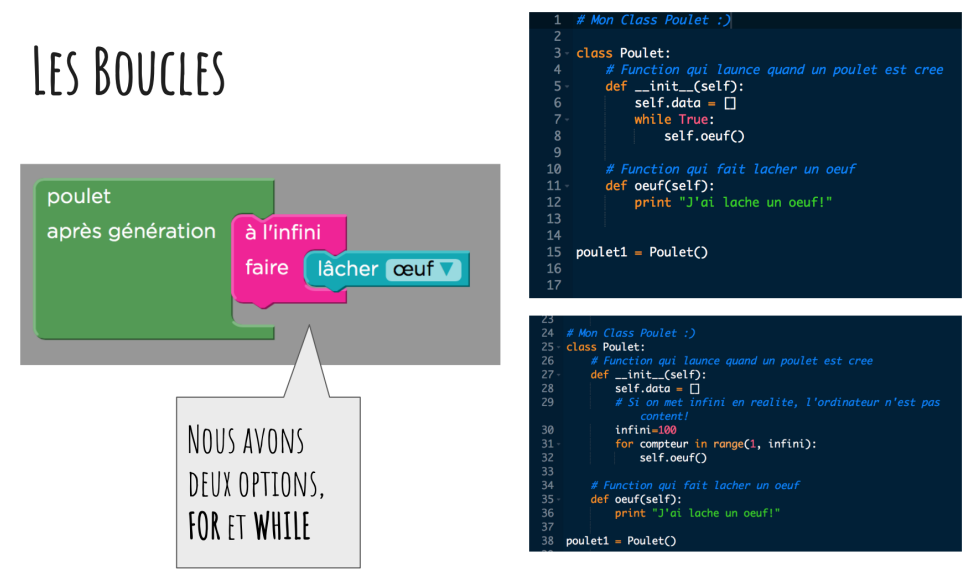

Figure 3. Loops explanation in French

objective is for the programmer to program the robot to follow a line on the floor (made with tape). Before starting, robot and programmer agree on instructions; a tap on the back means move forwards, a tap on the head means turn a quarter turn to the right. These are the only instructions that the robot knows. One agreed, the robot positions themselves at the beginning of the line, places a mask over their eyes and is programmed by the programmer. Challenges can include a left turn (i.e. turning right three times). Topics raised include precision (why is the robot not always on course?), and loops (could we make this more efficient by telling the robot to do the same sequence multiple times?).

Following the physical game, participants use an online, scratch-based platform to program more complex logic. We have used Code.org's Minecraft tutorial [12], but this could be replaced by any suitable alternative. Mentors are on hand to guide the participants, who should work in pairs (switching control of the computer periodically). Mentors are supplied with a booklet that contains example solutions and examples of how scratch code is translated to e.g. python as seen in Figure 3.

\section{A.2 What is a Website? (for ages 10-14)}

Despite using websites on a daily basis, much of the general public (adults and children alike) have not been exposed to the technology behind the web. In this workshop we introduce three web programming languages (CSS, HTML and Javascript) in a paper-based game and an online exercise to "fix" a broken website.

Participants listen to a brief introduction to the concept of the web and the three languages, with examples of sites that they know (in 2019 we used YouTube as a major example). Following that, participants work in pairs to draw a website on paper. One participant "fetches" a website (an envelope containing sheets of CSS, HTML and Javascript) from a "webserver" (a cardboard box), and they work together to decipher the size and colour of the divs and buttons.

Once finished, participants move to an online platform (currently we use Glitch [11]) that contains a broken website, which renders live on screen. Participants must change the colour, add their name and enable the Javascript on a button. For this workshop we developed a simple particle accelerator dashboard, to establish a link to CERN and science. Once working, 


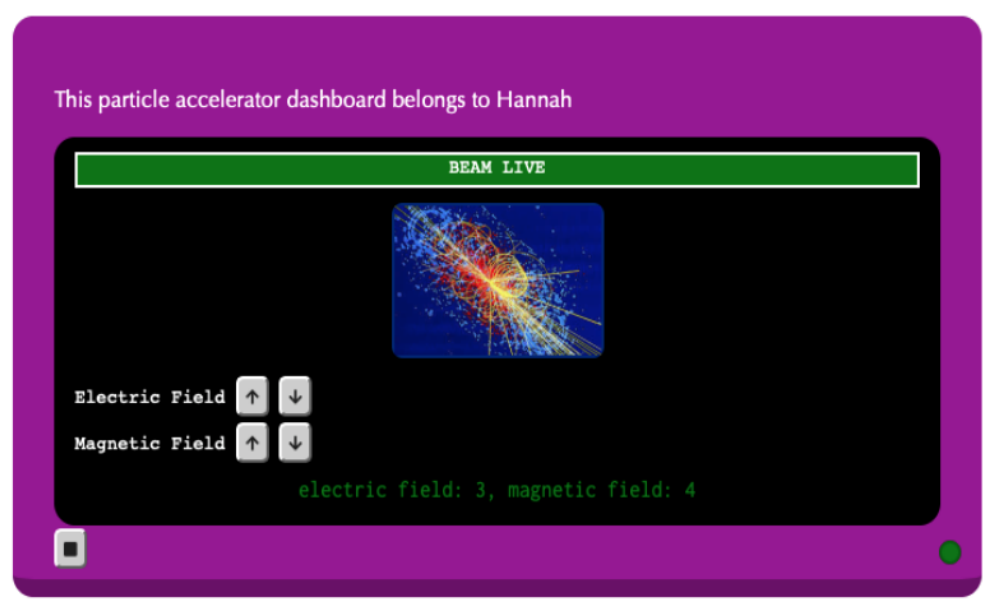

Figure 4. An example of a finished Website

the electric and magnetic fields can be manipulated to show an image of a collision as seen in Figure 4.

\section{A.3 Colouring Book (for younger children)}

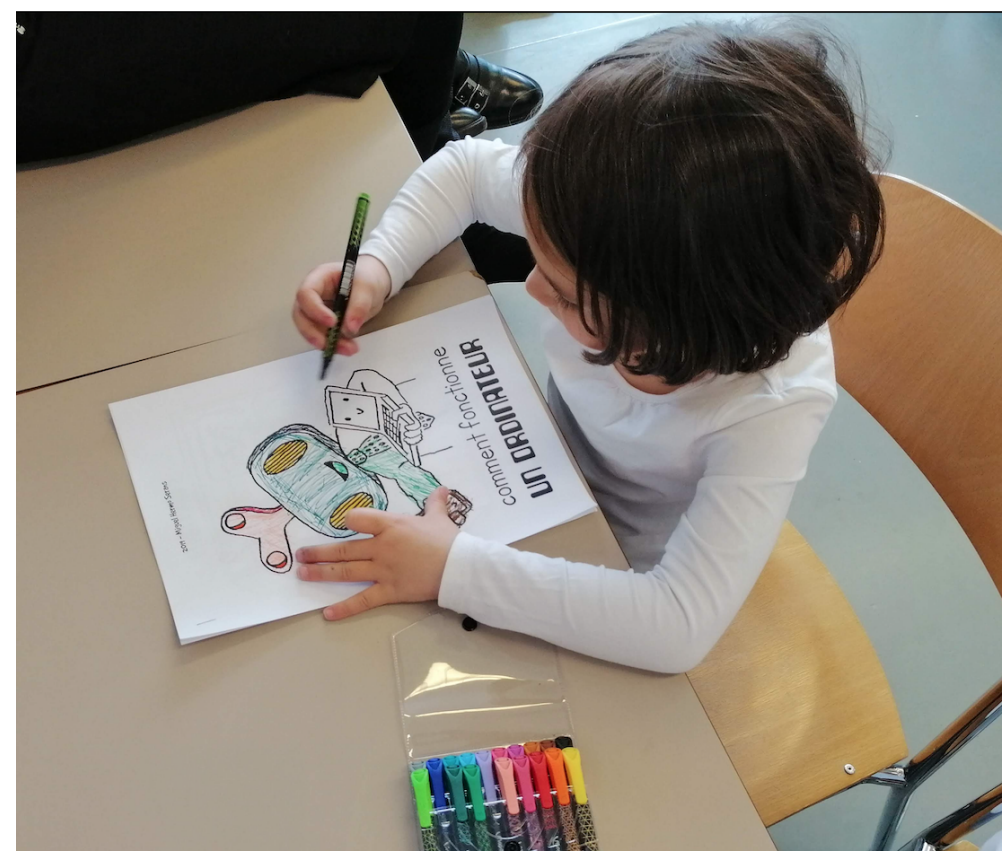

Figure 5. Colouring Book 
A simple colouring book is a useful resource for young participants (or younger siblings who may be waiting for a workshop to end). This colouring book introduces the concepts of input, memory, CPU and graphical processors using a robot, as seen in Figure 5, who calculates the value of $2+3$.

Many thanks go to all the volunteers who contributed to the Unplugged workshops during 2019, whose efforts totalled approximately 180 volunteer hours. The WIT Community is grateful for the support of these outreach activities from multiple bodies, including CERN's IT Department, the Local Engagement team and the Diversity and Inclusion Office.

\section{References}

[1] A. Gras-Velazquez, A. Joyce M. Debry, Women and ICT, Why are girls still not attracted to ICT studies and careers?, June 2009, http://eskills.eun.org

[2] CERN Annual Personnel Statistics, https://cds.cern.ch/collection/CERN\%20Annual\% 20Personnel\%20Statistics? $1 \mathrm{n}=\mathrm{en}$

[3] Neil Anderson, Colin Lankshear, Carolyn Timms, Lyn Courtney, 'Because it's boring, irrelevant and I don't like computers': Why high school girls avoid professionally-oriented ICT subjects, Computers Education, Volume 50, Issue 4, 2008, Pages 1304-1318, ISSN 0360-1315, https://doi.org/10.1016/j.compedu.2006.12.003.

[4] Vivian Hunt, Dennis Layton, Sara Prince, Why diversity matters, January 2015, https://www.mckinsey.com/ /media/McKinsey/Business\%20Functions/Organization/ Our\%20Insights/Why\%20diversity\%20matters/Why\%20diversity\%20matters.ashx

[5] Kate Brodock, Why we desperately need women to design AI, August 2017, https://www.freecodecamp.org/news/ why-we-desperately-need-women-to-design-ai-72cb061051df/

[6] Women in Technology at CERN, https://women-in-technology.web.cern.ch/about-us

[7] RightsTech Women, https://rightstech.org/about/

[8] Hello Ruby, https://www.helloruby.com/home

[9] 1,2,3, Codez, https://www.fondation-lamap.org/fr/123codez

[10] CERN Science Gateway, https://sciencegateway.cern

[11] Glitch, https://glitch.com

[12] Code.org, https://code.org

[13] Miliszewska, I., Moore, A. Moore, A. (2010). Encouraging Girls to Consider a Career in ICT: A Review of Strategies. Journal of Information Technology Education: Innovations in Practice, 9(1), 143-166. Informing Science Institute. Retrieved January 17, 2020 from https://www.learntechlib.org/p/111701/.

[14] Communications architecture: Vision, mission and themes, https://communications. web.cern.ch/strategy/communications-architecture-vision-mission-and-themes 